\title{
ANALYTIC TORSION AND R-TORSION FOR MANIFOLDS WITH BOUNDARY*
}

\author{
XIANZHE DAI ${ }^{\dagger}$ AND HAO FANG ${ }^{\ddagger}$
}

\begin{abstract}
We prove a formula relating the analytic torsion and Reidemeister torsion on manifolds with boundary in the general case when the metric is not necessarily a product near the boundary. The product case has been established by W. Luc̈k and S. M. Vishik. We find that the extra term that comes in here in the nonproduct case is the transgression of the Euler class in the even dimensional case and a slightly more mysterious term involving the second fundamental form of the boundary and the curvature tensor of the manifold in the odd dimensional case.
\end{abstract}

1. Introduction. The Reidemeister torsion (R-torsion for short) is a combinatorial/topological invariant associated to a unitary representation of the fundamental group of a manifold. Introduced by Reidemeister [R] and Franz [F], it is used to classify the lens spaces. It was further developed by Milnor and Whitehead and used successfully in classifying the cobordisms. In searching for an analytic interpretation of the R-torsion, Ray and Singer [RS1], [RS2] introduced the analytic torsion, which subsequently found many significant and far-reaching applications (Cf., for example, [B], [BGS], [BL], [GS], [DO], [W]).

The celebrated Cheeger-Müller theorem [C], [Mü1] establishes the Ray-Singer conjecture: namely, on closed manifolds, the analytic torsion is indeed the same as the R-torsion. Further significant work along this line includes that of Müller [Mü2] where he extends the theorem to unimodular representations, that of Bismut-Zhang [BZ], which treats general representations (in which interesting secondary invariants come in), and that of Burghelea-Friedlander-Kappeler-McDonald [BFKM] which deals with infinite dimensional representations (finite type Hilbert module).

All these work deals with closed manifolds. With appropriate boundary conditions, the torsion invariants can also be defined for manifolds with boundary. In fact, the torsions for manifolds with boundary provides an important stepping stone in Cheeger's approach to the Cheeger-Müller theorem (Cf. [C], [RS1]). In [C], the analog of Ray-Singer conjecture on manifolds with boundary is briefly discussed but the geometric information involved is not made explicit. This problem is addressed much later, first in Lott-Rothenberg [LR], and then Lück [L] and Vishik [V]. By assuming a product metric structure near the boundary, they found that the analytic torsion and the R-torsion differ by a topological invariant, namely, (up to a constant), the Euler number of the boundary. This work has been generalized by Burghelea-FriedlanderKappeler $[\mathrm{BFK}]$ to the finite type Hilbert module.

We obtain a general formula of the difference of analyitc torsion and Reidemaster torsion on a Riemannian manifold with boundary, without assuming the product metric structure near the boundary. In this case, there are two extra, geometric terms coming in, involving the second fundamental form of the boundary. In fact, one of the terms is the Chern-Simons class defined by Bismut-Zhang [BZ], transgressing the Euler class. This term therefore vanishes if the dimension of the manifold is odd. The other term, which vanishes for the even dimension, is only slightly more complicated,

*Received April 02, 1999; accepted for publication May 3, 1999.

†Department of Mathematics, University of California, Santa Barbara, Santa Barbara, CA 93106, U.S.A. (dai@math.ucsb.edu). Supported in part by NSF Grant DMS-9704296

$\ddagger$ Department of Mathematics, Princeton University, Princeton, NJ 08540, U.S.A. (haofang@ math.princeton.edu). 
involving the second fundamental form of the boundary and the curvature tensor of the manifold.

To be more precise, let $M$ be a compact Riemannian manifold with boundary $\partial M$ and metric $g=g^{T M}$. If we denote $x$ the geodesic distance to the boundary $\partial M$, then, near the boundary,

$$
g=d x^{2}+g_{\partial M}(x),
$$

where $g_{\partial M}(x)$ is a family of Riemannian metrics on the boundary. Let $g_{0}$ be a Riemannian metric which is of the form

$$
g_{0}=d x^{2}+g_{\partial M}(0)
$$

near the boundary. Let $\tilde{e}\left(g_{0}, g\right)$ be the Chern-Simons class defined in [BZ], which satisfies the equation

$$
d \tilde{e}\left(g_{0}, g\right)=e(g)-e\left(g_{0}\right),
$$

where $e(g)$ denotes the Pfaffian form of $g$. Set, for $l \in[0,1]$,

$$
g_{l}=l g+(1-l) g_{0}
$$

Let $\rho: \pi_{1}(M) \longrightarrow U(N)$ be a unitary representation. Denote by $i$ the inclusion of $\partial M$ into $M$.

THEOREM 1.1. Let $T=T(M, \rho)$ be the analytic torsion of $(M, \rho)$ with respect to the absolute boundary condition and $\tau=\tau(M, \rho)$ be the corresponding Reidemeister torsion. Then

$$
\ln (T / \tau)=\chi(\partial M, \rho) \ln 2+\operatorname{rank} \rho \int_{\partial M} i^{*} \tilde{e}\left(g_{0}, g\right)+c \operatorname{rank} \rho \int_{\partial M} i^{*} \phi,
$$

where

$$
c=\int_{0}^{\infty} \int_{0}^{1} \int_{0}^{\infty} x\left(e^{-\left(x-x^{\prime}\right)^{2} / 4 s}+e^{-\left(x+x^{\prime}\right)^{2} / 4 s}\right) 2 e^{-\left(x+x^{\prime}\right)^{2} / 4(1-s)} d x^{\prime} d s d x
$$

and $\phi$ is a differential form on $M$ defined by the Berezin integral

$$
\int^{B} \sum h_{a b} e^{a} \wedge \hat{e}^{b} \mathcal{R}_{0}^{\prime} e^{-\mathcal{R}} d l
$$

where $h_{a b}, 1 \leq a, b \leq n-1$, is the second fundamental form of the boundary,

$$
\mathcal{R}_{0}^{\prime}=\frac{1}{4} R_{0 j k l} e^{j} \wedge \hat{e}^{k} \wedge \hat{e}^{l}
$$

and

$$
\mathcal{R}=\frac{1}{8} R_{i j k l} e^{i} \wedge e^{j} \wedge \hat{e}^{k} \wedge \hat{e}^{l} .
$$

REMARK. One also obtain a similar formula for the relative boundary condition, with only sign changes. 
REMARK. The reader should consult [LS] for examples where the these geometric contributions are shown to be nonzero, using the formulas given in [BG]. The article $[\mathrm{BG}]$ also contains a thorough discussion on the aboslute and relative boundary conditions.

An immediate application of this result is a gluing formula for the analytic torsion, since the gluing formula for R-torsion is well known.

Our strategy of proof is a very natural one. Namely we deform to the product case, where the difference of the analytic torsion and R-torsion is known by [L], [V]. The variation of the $\log$ of the ratio of the analytic torsion over the $\mathrm{R}$-torsion is given by the constant term in certain asymptotic expansion of the heat kernel. To compute this term we employ the local index theorem technique, much in the spirit of BismutZhang [BZ]. The new difficulty, however, is that the boundary condition introduces certain non-uniform behavior in the local heat asymptotic expansion, in the form of some Gaussian type concentration near the boundary. This difficulty is overcome by bringing in the technique of Melrose (Cf. [DM]). The non-uniform behavior of the heat kernel for the absolute boundary condition (say) is resolved by lifting it to a larger space obtained by performing certain blowup operation on the usual carrier of the heat kernel. This technique also effectively separates the interior and boundary contributions. The interior contribution is zero, just as in the closed case, while the boundary contribution comes from a model problem on the half tangent space of the manifold at the boundary.

ACKnowledgment. The authors are very grateful to Xiaonan Ma for his careful reading of an earlier version and for pointing out several mistakes and misprints. The authors would like to thank Jean-Michel Bismut, Alice Chang, Paul Yang and Weiping Zhang for their interests and useful discussion. The second author would also like to thank Alice Chang, Paul Yang and Weiping Zhang for their constant encouragement.

2. Analytic torsion for manifold with boundary. In this section we recall the definition of the absolute and relative boundary conditions, their corresponding torsion invariants, while fixing our notations. We also state the variational formula for the log of the ratio of the analytic torsion over the R-torsion (from [RS1] and [C]), which is our starting point.

2.1. Absolute and relative boundary conditions. Let $M$ be a compact manifold with boundary and $g$ be a Riemannian metric on $M$. If we denote $x$ the geodesic distance to the boundary $\partial M$, then, near the boundary,

$$
g=d x^{2}+g_{\partial M}(x)
$$

where $g_{\partial M}(x)$ is a family of Riemannian metrics on the boundary. Let $\xi \rightarrow M$ be the flat bundle associated to a representation $\rho: \pi_{1}(M) \rightarrow U(k)$. Consider the Hodge Laplacian

$$
\Delta=(d+\delta)^{2}: C^{\infty}(M ; \Lambda(M) \otimes \xi) \rightarrow C^{\infty}(M ; \Lambda(M) \otimes \xi)
$$

At the boundary we have the splitting

$$
\left.\Lambda(M) \otimes \xi\right|_{\partial M}=\Lambda(\partial M) \otimes \xi \oplus \Lambda(\partial M) \otimes \xi
$$

corresponding to the decomposition for a form $\omega \in C^{\infty}(M ; \Lambda(M) \otimes \xi)$ :

$$
\omega=\omega_{t}+d x \wedge \omega_{n}, \omega_{t}, \omega_{n} \in C^{\infty}(M ; \Lambda(\partial M) \otimes \xi)
$$


near the boundary. Define a linear map $\sigma$ :

$$
\sigma(\omega)=\omega_{t}-d x \wedge \omega_{n}
$$

Then $\sigma$ is self adjoint and $\sigma^{2}=1$. Moreover the splitting (2.2) corresponds to the decomposition into the \pm 1 -eigenspace of $\sigma$.

From the splitting we define two projections

$$
\begin{aligned}
P_{a}: C^{\infty}\left(\partial M ;\left.\Lambda(M) \otimes \xi\right|_{\partial M}\right) & \rightarrow C^{\infty}(\partial M ; \Lambda(\partial M) \otimes \xi), \\
P_{a}(\omega) & =\left.\omega_{n}\right|_{\partial M} ; \\
P_{r}: C^{\infty}\left(\partial M ;\left.\Lambda(M) \otimes \xi\right|_{\partial M}\right) & \rightarrow C^{\infty}(\partial M ; \Lambda(\partial M) \otimes \xi), \\
P_{r}(\omega) & =\left.\omega_{t}\right|_{\partial M} .
\end{aligned}
$$

i.e., $P_{a}$ is the orthogonal projection onto the -1-eigenspace of $\sigma$ and $P_{r}$ the orthogonal projection onto the +1 -eigenspace. The absolute and relative boundary conditions for the Hodge Laplacian are

$$
\begin{gathered}
\text { absolute }:\left\{\begin{array}{l}
P_{a}\left(\left.\omega\right|_{\partial M}\right)=0 \\
P_{a}\left(\left.\omega\right|_{\partial M}\right)=0
\end{array}\right. \\
\text { relative }:\left\{\begin{array}{l}
P_{r}\left(\left.\omega\right|_{\partial M}\right)=0 \\
P_{r}\left(\left.\delta \omega\right|_{\partial M}\right)=0
\end{array}\right.
\end{gathered}
$$

It is well known that these define elliptic boundary conditions for the Hodge Laplacian, with the corresponding cohomology being the absolute (resp. relative) cohomology of $M$. Hence the names.

To get a flavor of these boundary conditions, the special case when $M=\partial M \times \mathbb{R}_{4}$, and $g=d x^{2}+g_{\partial M}$, where $x$ is the variable of $\mathbb{R}_{+}$and $g_{\partial M}$ is a metric on the boundary, deserves some elaboration. Using separation of variables, we can write

$$
\omega=f(x) \omega_{1}^{\partial M}+g(x) d x \wedge \omega_{2}^{\partial M}
$$

where $\omega_{1}^{\partial M}$ and $\omega_{2}^{\partial M}$ are differential forms on $\partial M$. Then the absolute and relative boundary conditions are reduced to a combination of Dirichlet and Neumann boundary conditions:

$$
\begin{gathered}
\text { absolute }:\left\{\begin{array}{l}
g(0)=0 \\
\frac{\partial f}{\partial x}(0)=0
\end{array}\right. \\
\text { relative : }\left\{\begin{array}{l}
f(0)=0, \\
\frac{\partial g}{\partial x}(0)=0
\end{array}\right.
\end{gathered}
$$

This greatly simplifies our computation of the model problem.

Back to the general discussion, it follows easily from the Stokes' Theorem that

$$
(\Delta \omega, \theta)=(\omega, \Delta \theta)+\int_{\partial M} \omega \wedge * d \theta-\int_{\partial M} \theta \wedge * d \omega+\int_{\partial M} d^{*} \omega \wedge * \theta-\int_{\partial M} d^{*} \theta \wedge * \omega
$$

The boundary terms all vanish if both $\omega, \theta$ satisfy the absolute (resp. relative) boundary condition. i.e. the absolute and relative boundary conditions are also self adjoint boundary conditions. 
Therefore, if $F_{1}(t, x, z), F_{2}(t, z, y)$ are double forms on $M$ that both satisfy the absolute (resp. relative) boundary condition, then (Cf. [C, p275])

$$
\begin{aligned}
& \left(F_{1}(0, x, \cdot), F_{2}(t, \cdot, y)\right)-\left(F_{1}(t, x, \cdot), F_{2}(0, \cdot, y)\right) \\
& =\int_{0}^{t}\left(F_{1}(t-s, x, \cdot),\left(\partial_{t}+\Delta\right) F_{2}(s, \cdot, y)\right) d s-\int_{0}^{t}\left(\left(\partial_{t}+\Delta\right) F_{1}(t-s, x, \cdot), F_{2}(s, \cdot, y)\right) d s .
\end{aligned}
$$

Thus if we denote

$$
F_{1} * F_{2}=\int_{0}^{t} \int_{M}\left\langle F_{1}(t-s, x, z), F_{2}(s, z, y)\right\rangle d z d s
$$

then we have arrived at the so called Duhamel principle:

Proposition 2.1. Let $e^{-t \Delta}$ denote the heat kernel of the Laplacian with the absolute (resp. relative) boundary condition. Let $F_{1}(t, x, y)$ be a double form on $M$ which satisfies the absolute (resp. relative) boundary condition, and is the delta function at $t=0$. Then

$$
e^{-t \Delta}=\sum_{m=1}^{\infty}(-1)^{m-1} F_{m}
$$

where $F_{m}=F_{m-1} *\left(\partial_{t}+\Delta\right) F_{1}$.

Proof. This follows by iterating the formula above.

2.2. Analytic torsion and R-torsion. With elliptic self adjoint boundary conditions at our disposal the analytic torsions for manifolds with boundary can be defined using exactly the same formula as in the closed case. We only need to replace the usual heat kernel with the heat kernel corresponding to the appropriate boundary conditions. For simplicity it will be implicitly assumed that our heat kernels correspond to the absolute boundary condition, unless stated otherwise.

Thus, the analytic torsion $T(M, \rho)$ is defined in terms of the torsion zeta function

$$
\log T(M, \rho)=\zeta_{T}^{\prime}(0)
$$

where $\zeta_{T}(s)$ is a meromorphic function of $s \in \mathbb{C}$, defined as follows. For $\Re s>>0$

$$
\zeta_{T}(s)=\frac{1}{\Gamma(s)} \sum_{p=1}^{\operatorname{dim} M} p(-1)^{p+1} \int_{0}^{\infty} t^{s} \operatorname{Tr}\left(e^{-t \Delta_{p}} P^{\perp}\right) \frac{d t}{t}
$$

where $\Delta_{p}$ is the Laplacian on $p$-forms satisfying the absolute boundary conditions, and $P^{\perp}$ denote the projection onto the orthogonal complement of the harmonic forms. Using the standard elliptic theory, one sees that $\zeta_{T}(s)$ extends to a meromorphic function of $s \in \mathbb{C}$ which is regular at $s=0$. In particular, the right hand side of (2.9) is well-defined.

The natural $\mathbb{Z}$ grading on the space of differential forms induces a natural $\mathbb{Z}_{2}$ grading. We denote the corresponding supertrace $\operatorname{Tr}_{s}$. With this notation formula (2.10) can be rewritten as

$$
\zeta_{T}(s)=-\frac{1}{\Gamma(s)} \int_{0}^{\infty} t^{s} \operatorname{Tr}_{s}\left(N e^{-t \Delta} P^{\perp}\right) \frac{d t}{t}
$$


where $N$ denotes the so called number operator which mutiplies a differential $p$-form by $p$.

Similarly, the corresponding R-torsion $\tau(M, \rho)$ can be defined the same way as in the closed case using the absolute cochain complex.

The analytic torsion as defined above depends on the Riemannian metric $g$. So does the R-torsion. The variation of the torsion invariants with respect to the metric change is well studied. We quote the following result from [RS1], [C].

Proposition 2.2. Let $g_{l}$ be a family of Riemannian metrics $(l \in[0,1])$, and $*_{l}$ the corresponding Hodge *-operator. Denote $T_{l}=T_{l}(M, \rho)\left(\right.$ resp. $\left.\tau_{l}=\tau_{l}(M, \rho)\right)$ the analytic torsion (resp. $R$-torsion) with respect to $g_{l}$. If the unit normal of $\partial M$ with respect to $g_{l}$ is independent of the parameter $l$, then

$$
\frac{d}{d l} \log T_{l} / \tau_{l}=\lim _{t \rightarrow 0} \operatorname{Tr}_{s}\left(*_{l}^{-1} \frac{d *_{l}}{d l} e^{-t \Delta_{l}}\right)
$$

REMARK. In general the right hand side of (2.11) should be interpreted as the constant term in the asymptotic expansion of $\operatorname{Tr}_{s}\left(*_{l}^{-1} \frac{d *_{l}}{d l} e^{-t \Delta_{l}}\right)$. In our case, however, all the negative powers of the asymptotic expansion drop out, as we will see from our proof, and the limit therefore is the genuine limit.

Thus, to prove our result, we deform to the product case. Namely, if we denote $x$ the geodesic distance to the boundary $\partial M$ with respect to the Riemannain metric $g$ on $M$, then $g$ can be written as

$$
g=d x^{2}+g_{\partial M}(x)
$$

near the boundary. Now, let $g_{0}$ be a Riemannian metric which is of the form

$$
g_{0}=d x^{2}+g_{\partial M}(0)
$$

near the boundary. Set

$$
g_{l}=l g+(1-l) g_{0} .
$$

We compute the right hand side of (2.11), using the local index theorem technique in the spirit of [BZ]. However, before that is possible, we first need to develop a thorough understanding of the uniform structure of the heat kernel for absolute (or relative) boundary condition.

3. The Uniform Structure of Heat Kernel. In this section we analyze the uniform structure of the heat kernel for elliptic boundary value problems of the type discussed above. The local index theorem technique dictates that we study the pointwise trace of the heat kernel. However, the elliptic boundary conditions introduce certain nonuniform behavior in the pointwise trace of the heat kernel in the form of some Gaussian type concentration at the boundary. We resolve this nonuniform behavior by studying the heat kernel in a bigger space, by introducing certain singular coordinates. In fact, we will construct a pseudodifferential calculus adapted to the situation and show that the heat kernel is certain element in this calculus. All the regularity and uniform property is contained in this statement.

3.1. Construction of the pseudodifferential calculus. Let $Z=M \times M \times \mathbb{R}_{+}$ be the usual carrier for the heat kernel. If we denote by $(x, y)$ the local coordinates of $M$ near $\partial M$, then $\left(x, y, x^{\prime}, y^{\prime}, t\right)$ will be a local coordinates on $Z$. Our analysis of the 
heat kernel will be carried out in a blowup version of the space $Z$. For a space $X$, we denote by $\Delta(X)$ the diagonal in $X \times X$. Let $B_{1}=\Delta(\partial M) \times\{0\}$ and $B_{2}=\Delta(M) \times\{0\}$ be subspaces of $Z$. Also denote by $S$ the parabolic bundle generated by $d t$. Consider the space $[\mathrm{DM}]$

$$
M_{h 0}^{2}=\left[Z ; B_{1}, B_{2}, S\right]
$$

obtained from $Z$ by blowing up, parabolically in $t$, first $B_{1}$, then $B_{2}$. The blow down map is denoted by $\beta_{h 0}$. That is, $\beta_{h 0}: M_{h 0}^{2} \rightarrow Z$.

The new space $M_{h 0}^{2}$ is a manifold with corner. For the moment let's look at the structures of the boundary hypersurfaces. There are three of them lying above $\{t=0\}$. Among these we have $B_{f f}$ from the blow up of $B_{1}$, called the front face; $B_{t f}$ from the blow up of $B_{2}$, called the temporal face. These two are fibered over the submanifolds that are blown up. In fact $B_{t f}$ can be viewed as a natural compactification of the rescaled tangent bundle ${ }^{0} T M$ of $M$, whose sections are precisely vector fields vanishing at the boundary, as can be verified using suitable coordinates such as (3.4). Similarly $B_{f f}$ is related to the half tangent bundle of $M$ at $\partial M$ as follows: the front face $B_{f f}^{\prime}$ resulting from the first blowup fibers over $B_{1} \cong \partial M$, and in fact is canonically isomorphic to

$$
\mathbb{R}_{+} \times T_{\partial M}^{+} M \rightarrow \partial M
$$

with $T_{\partial M}^{+} M$ denoting the half tangent bundle of $M$ at $\partial M$. Then $B_{f f}$ is a suitable blowup of $B_{f f}^{\prime}$, namely, if $T$ is the coordinate of $\mathbb{R}_{+},\left(s, \bar{Y}, y^{\prime}\right)$ the fiber coordinates of $T_{\partial M}^{+} M$, then $B_{f f}$ is obtained by fiberwise blowing up $T=0, s=1, \bar{Y}=0$.

The rest of the boundary hypersurfaces arises from the lift of those of $Z$ under the blowdown map. More precisely we have the lift of the hypersurface $t=0$ of $Z$, denoted by $B_{t b}\left(M_{h 0}^{2}\right)$; the lift of the hypersurface $x=0$ of $Z, B_{l b}\left(M_{h 0}^{2}\right)$; and the lift of $x^{\prime}=0, B_{r b}\left(M_{h 0}^{2}\right)$. Note that $B_{t f}$ only meets $B_{f f}, B_{t b}$.

In effect, the blowup is equivalent to introducing certain singular coordinates near the boundary. In the first blowup, the projective coordinates

$$
T=t / x^{\prime 2}, s=x / x^{\prime}, x^{\prime}, \bar{Y}=\frac{y-y^{\prime}}{x^{\prime}}, y^{\prime}
$$

will be valid coordinates near the front face, except at the corner with $B_{r b}$. After the second blowup, we can use the projective coordinates

$$
T, X=\frac{s-1}{\sqrt{T}}=\frac{x-x^{\prime}}{\sqrt{t}}, Y=\frac{\bar{Y}}{\sqrt{T}}=\frac{y-y^{\prime}}{\sqrt{t}}, x^{\prime}, y^{\prime}
$$

near the temporal face, except at the corner with $B_{t b}$.

We will denote by $\rho$ the defining function for each boundary hypersurface, with the appropriate subscript attached. Thus $\rho_{f f}$ will denote the defining function of $B_{f f}$, etc.

The pseudodifferential calculus will consists of operators whose kernels are defined on the space $M_{h 0}^{2}$ and normalized with respect to the half-density

$$
K D_{h 0}=\rho_{f f}^{-(n+2) / 2} \rho_{t f}^{-(n+3) / 2} \Omega^{1 / 2}\left(M_{h 0}^{2}\right),
$$

where $\Omega^{1 / 2}$ denotes the standard half-density bundle. Let $I$ denote the index set $\left\{k_{1}, k_{2}, k_{3}, k_{4}\right\}$. The space of operators with index set $I$ is defined to be

$$
\Psi_{h 0}^{I}(M, \Lambda M \otimes \xi)=\rho_{f f}^{k_{1}} \rho_{t f}^{k_{2}} \rho_{l b}^{k_{3}} \rho_{r b}^{k_{4}} \rho_{t b}^{\infty} C^{\infty}\left(M_{h 0}^{2} ; \operatorname{Hom}(\Lambda M \otimes \xi) \otimes K D_{h 0}\right) .
$$


To see how the operators in $\Psi_{h 0}^{I}$ act, consider the bilinear map from

$$
\dot{C}_{0}^{\infty}\left([0, \infty) \times M ; \Lambda M \otimes \xi \otimes \Omega^{1 / 2}\right) \times \dot{C}^{\infty}\left([0, \infty) \times M ; \Lambda M \otimes \xi \otimes \Omega^{1 / 2}\right)
$$

to

$$
\dot{C}^{\infty}\left(Z ; \Lambda M \otimes \xi \otimes \Omega^{1 / 2}\right)
$$

defined by

$$
\phi \hat{*}_{t} \psi=\int_{0}^{\infty} \phi\left(t+t^{\prime}, x\right) \psi\left(t^{\prime}, x^{\prime}\right) d t^{\prime}
$$

Lifting to $M_{h 0}^{2}$ we can define

$$
\langle A \psi, \phi\rangle=\int_{M_{h 0}^{2}} A \cdot \beta_{h 0}^{*}\left(\phi \hat{*}_{t} \psi\right) .
$$

It can be shown that these operators form a filtered algebra containing the heat kernel of an elliptic boundary value problem. However we do not need the full strength of this result and therefore will only be establishing those that we need in the following subsections.

3.2. Normal homomorphisms. Just like the classical pseudodifferential operators, the regularity property of elements of $\Psi_{h 0}^{I}$ is described by their symbols, except that there are more than just one (principal) symbol here, called the normal homomorphisms.

The normal homomorphism at $B_{t f}$, or the heat homomorphism, is defined by dividing out $T^{\frac{k_{2}}{2}}$ and restricting to $B_{t f}$ :

$$
N_{h, k_{2}}: \Psi_{h 0}^{I} \rightarrow \rho_{f f}^{k_{1}} \rho_{l b}^{k_{3}} \rho_{r b}^{k_{4}} \rho_{t b}^{\infty} C^{\infty}\left(B_{t f} ;\left.K D_{h 0}\right|_{B_{t f}}\right) .
$$

Since elements of $\Psi_{h 0}^{I}$ vanish rapidly at $B_{t b}$ the heat homomorphism simply describes the leading term at $B_{t f}$. We note that $B_{t f}$ meets only $B_{f f}$ and $B_{t b}$. Moreover, since $B_{t f}$ is the natural compactification of ${ }^{0} T M,\left.K D_{h 0}\right|_{B_{t f}}$ is canonically isomorphic to the fiber density bundle of ${ }^{0} T M$ and thus the heat homomorphism can be rewritten as

$$
N_{h, k_{2}}: \Psi_{h 0}^{I} \rightarrow \rho_{f f}^{k_{1}} \mathcal{S}\left({ }^{0} T M ; \Omega_{\text {fiber }}\right) .
$$

Here $\mathcal{S}\left({ }^{0} T M ; \Omega_{\text {fiber }}\right)$ denotes the space of smooth fiber densities which are of Schwarz class along fibers of ${ }^{0} T M$.

The normal homomorphism at $B_{f f}$ describes the leading term at $B_{f f}$. Thus we divide out $\rho_{f f}^{k_{1}}$ and restrict to $B_{f f}$ :

$$
N_{f, k_{1}}: \Psi_{h 0}^{I} \rightarrow \rho_{t f}^{k_{2}} \rho_{l b}^{k_{3}} \rho_{r b}^{k_{4}} \rho_{t b}^{\infty} C^{\infty}\left(B_{f f} ;\left.K D_{h 0}\right|_{B_{f f}}\right)
$$

By the previous discussion on the structure of $B_{f f}$ (see (3.2)) the range of $N_{f, k_{1}}$ is isomorphic to the space of smooth functions on $\mathbb{R}_{+} \times T_{\partial M}^{+} M$, except for conormal concentration along the submanifold $\dot{s}=1, \bar{Y}=0$ at $T=0$, with the order of vanishing at the boundaries $s=0$ and $s=\infty$ prescribed by the index set, and 
vanishing rapidly at $\bar{Y}$-infinity. In other words, the range of $N_{f, k_{1}}$ is the (restricted) fiberwise heat calculus on $T_{\partial M}^{+} M$.

For an operator in $\Psi_{h 0}^{I}$, its values under the normal homomorphisms will be called its normal operators. Individually, each normal homomorphism is surjective. However the normal operators for an element of $\Psi_{h 0}^{I}$ have to agree at the common corners. These are the compatibility conditions. On the other hand, since essentially just smooth functions are involved, the compatibility conditions are clearly the only obstructions to the existence of operators with the prescribed normal operators. This fact will be used in constructing heat kernels as an element of $\Psi_{h 0}^{I}$.

3.3. Compositions. Another ingredient in constructing heat kernels as an element of $\Psi_{h 0}$ will be the composition properties of elements of $\Psi_{h 0}$. We first study the composition of these operators with differential operators.

Proposition 3.1. Let $A \in \Psi_{h 0}^{I}(M, \Lambda M \otimes \xi)$. If $V$ is any smooth vector field on $M$, and $T=t / x^{\prime 2}$, then we have

$$
\left(t^{\frac{1}{2}} V\right) \circ A \in \Psi_{h 0}^{I}(M, \Lambda M \otimes \xi)
$$

and

$$
N_{f, k_{1}}\left(t^{\frac{1}{2}} V \circ A\right)=T^{\frac{1}{2}} \sigma_{1}(V) N_{f, k_{1}}(A), \quad N_{h, k_{2}}\left(t^{\frac{1}{2}} V \circ A\right)=\sigma_{1}(V) N_{h, k_{1}}(A) .
$$

Here $\sigma_{1}(V)$ denotes the usual symbol of a vector field. Furthermore,

$$
\left(t \partial_{t} \circ A\right) \in \Psi_{h 0}^{I}(M, \Lambda M \otimes \xi)
$$

and

$(3.11) N_{f, k_{1}}\left(t \partial_{t} \circ A\right)=T \partial_{T} N_{f, k_{1}}(A), N_{h, k_{2}}\left(t \partial_{t} \circ A\right)=-\frac{1}{2}\left(R+n+2+k_{2}\right) N_{h, k_{2}}(A)$.

Proof. We first assume that $\rho$ is the trivial representation, i.e. $\xi$ is the trivial bundle. Clearly we can restrict our attention to a region near the front and temporal faces. Therefore we assume that $A$ is supported near such a region. Further, by partition of unity, we can assume that $A$ is either supported near the front face but away from the temporal face, or is supported near the temporal face.

For the first case, we get to use the projective coordinates (3.3), But it is more convenient to use the project coordinate

$$
t, X=\frac{x}{t^{\frac{1}{2}}}, X^{\prime}=\frac{x^{\prime}}{t^{\frac{1}{2}}}, Y=\frac{y-y^{\prime}}{t^{\frac{1}{2}}}, y^{\prime},
$$

which is valid near the front face, except at the corner, where $X, X^{\prime}, Y=\infty$. In this coordinate $A \in \Psi_{h 0}^{I}(M)$ can be written as $A=t^{\frac{k_{1}}{2}-\frac{n+2}{4}} a\left(t, X, X^{\prime}, Y, y^{\prime}\right)\left|d t d X d X^{\prime} d Y d y^{\prime}\right|^{\frac{1}{2}}$, where $a$ is a smooth function of $\left(t^{\frac{1}{2}}, X, X^{\prime}, Y, y^{\prime}\right)$ and vanishes rapidly as $X, X^{\prime}, Y \rightarrow$ $\infty$. In this representation $N_{f, k_{1}}(A)=a\left(0, X, X^{\prime}, Y, y^{\prime}\right)\left|d X d X^{\prime} d Y d y^{\prime}\right|^{\frac{1}{2}}$.

Now if $\phi=\phi_{0}|d t d x d y|^{\frac{1}{2}}$ and $\psi=\psi_{0}|d t d x d y|^{\frac{1}{2}}$ we have

$$
\langle A \psi, \phi\rangle=\int_{M_{h}^{2}} t^{\frac{k_{1}}{2}} a \beta_{h}^{*}\left(\phi_{0} \hat{*}_{t} \psi_{0}\right)\left|d t d X d X^{\prime} d Y d y^{\prime}\right| .
$$

Also, if we let $V=v_{1}(x, y) \partial_{x}+v_{2}(x, y) \partial_{y}$ be a smooth vector field and $V^{\prime}$ denote its transpose:

$$
\langle V \psi, \phi\rangle=-\left\langle\psi, V^{\prime} \phi\right\rangle
$$


then $V^{\prime}=\partial_{x} \circ v_{1}+\partial_{y} \circ v_{2}$, and $\beta_{h}^{*}\left(t^{\frac{1}{2}} V^{\prime}\right)=t^{\frac{1}{2}} \partial_{x}\left(v_{1}\right)+t^{\frac{1}{2}} \partial_{y}\left(v_{2}\right)+v_{1} \partial_{X}+v_{2} \partial_{Y}$.

A computation similar to that of [DM, p44] then shows that

$\left(t^{\frac{1}{2}} V\right) \circ A=t^{\frac{k_{1}}{2}-\frac{n+2}{4}}\left(v_{1} \partial_{X}(a)+v_{2} \partial_{Y}(a)+t^{\frac{1}{2}}\left(\partial_{x} v_{1}\right) a+t^{\frac{1}{2}}\left(\partial_{y} v_{2}\right) a\right)\left|d t d X d X^{\prime} d Y d y^{\prime}\right|^{\frac{1}{2}}$

and therefore $\left(t^{\frac{1}{2}} V\right) \circ A \in \Psi_{h 0}^{I}(M)$, with

$$
N_{f, k_{1}}\left(t^{\frac{1}{2}} V \circ A\right)=\left(v_{1} \partial_{X}+v_{2} \partial_{Y}\right) a\left(0, X, X^{\prime}, Y, y^{\prime}\right)\left|d X d X^{\prime} d Y d y^{\prime}\right|^{\frac{1}{2}}
$$

Now we change the coordinate from (3.12) to (3.3) and get

$$
\partial_{X}=T^{\frac{1}{2}} \partial_{s}, \quad \partial_{Y}=T^{\frac{1}{2}} \partial_{\bar{Y}},
$$

which, when plugged into (3.13), immediately gives

$$
N_{f, k_{1}}\left(t^{\frac{1}{2}} V \circ A\right)=T^{\frac{1}{2}} \sigma_{1}(V) N_{f, k_{1}}(A) .
$$

Similarly we have $t \partial_{t} \circ A \in \Psi_{h 0}^{I}(M)$ and

$$
N_{f, k_{1}}\left(t \partial_{t} \circ A\right)=\left[\frac{k_{1}}{2}-\frac{1}{2}\left(n+1+X \partial_{X}+X^{\prime} \partial_{X}^{\prime}+Y \partial_{Y}\right)\right] N_{h,-j}(A) .
$$

But changing to the coordinate (3.3) we see

$$
N_{f, k_{1}}\left(t \partial_{t} \circ A\right)=T \partial_{T} N_{f, k_{1}}(A) .
$$

For the second case when the support of $A$ is near the temporal face, the normal operators can be obtained in the same way using the projective coordinates (3.4).

Finally, if $\rho$ is not trivial, by linearity, we can assume that $\phi, \psi$ and $A$ are supported in a small neighborhood where $\xi$ is trivialized by an orthonormal basis $\left\{s_{i}\right\}$. Write

$$
\phi=\phi_{i} s_{i}, \psi=\psi_{i} s_{i}, A=A_{i j} s_{i}^{*} \otimes s_{j}
$$

Then

$$
\begin{aligned}
\langle(V \circ A) \psi, \phi\rangle & =-\left\langle A \psi, \nabla_{V} \phi\right\rangle \\
& =-\left\langle A_{i j} \psi_{i}, V \phi_{j}+\Gamma_{k j}(V) \phi_{k}\right\rangle,
\end{aligned}
$$

where $\Gamma_{k j}(V)=\left\langle\nabla_{V} s_{i}, s_{j}\right\rangle$. This reduces to the scalar case and the connection produces only a lower order term. $\square$

We now consider the composition of elements of $\Psi_{h 0}^{I}(M ; \Lambda M \otimes \xi)$. For simplicity we will not consider the full composition properties, only the composition of the following Voltera type operators, which suffices for our purpose. Thus if $\mathcal{E}=\left(E_{f f}, E_{l b}, E_{r b}\right)$ is an index family for $M_{h 0}^{2}$, assumed trivial at $B_{t f}$ and $B_{t b}$ then let

$$
\Psi_{h 0}^{-\infty, \mathcal{E}}\left(M, \Omega^{\frac{1}{2}}\right)=\Psi_{h 0}^{E_{f f}, \infty, E_{l b}, E_{r b}}(M, \Lambda M \otimes \xi)
$$

be the space of polyhomogeneous conormal distributions on $M_{h 0}^{2}$ which vanish rapidly at $B_{t f}$ and $B_{t b}$ and have expansions at $B_{f f}, B_{l b}$ and $B_{r b}$ with exponents from $E_{f f}$, $E_{l b}$ and $E_{r b}$ respectively.

Proposition 3.2. We have

$$
\Psi_{h 0}^{-\infty, \mathcal{E}}\left(M, \Omega^{\frac{1}{2}}\right) \circ \Psi_{h 0}^{-\infty, \mathcal{F}}\left(M, \Omega^{\frac{1}{2}}\right) \subset \Psi_{h 0}^{-\infty, \mathcal{G}}\left(M, \Omega^{\frac{1}{2}}\right)
$$


where

$$
\begin{aligned}
G_{f f} & =\left[E_{f f}+F_{f f}\right] \cup\left[E_{l b}+F_{r b}+n+2\right] \\
G_{l b} & =\left[E_{f f}+F_{l b}\right] \cup\left[E_{l b}\right] \\
G_{r b} & =\left[F_{f f}+E_{r b}\right] \cup\left[F_{r b}\right] .
\end{aligned}
$$

Proof. Since the operators here all have their Schwartz kernel vanishing rapidly at $B_{t f}$, we might as well consider them as living in

$$
M_{0}^{2}=\left[Z ; B_{1}, S\right] \text {. }
$$

The proof relies on lifting everything to a triple space. For this reason we consider

$$
W=\mathbb{R}_{+}^{2} \times M^{3}=\left\{\left(t, t^{\prime}, m, m^{\prime}, m^{\prime \prime}\right) \mid t \geq t^{\prime}\right\},
$$

and the following submanifolds of $W$ :

$$
B_{F}=\left\{\left(t, 0, m, m^{\prime}, m^{\prime}\right)\right\}, \quad B_{S}==\left\{\left(t, t, m, m, m^{\prime \prime}\right)\right\}, \quad B_{C}=\left\{\left(0, t^{\prime}, m, m^{\prime}, m\right)\right\},
$$

together with

$$
B_{T}=\{(0,0, m, m, m)\},
$$

which is the intersection of the all three. Let $S_{F}, S_{S}, S_{C}$ be the lift of the parabolic bundle $S=\{d t\}$ to the corresponding submanifolds $B_{F}, B_{S}, B_{C}$ and $S_{T}=\left\{d t, d t^{\prime}\right\}$ the parabolic bundle on $B_{T}$. Then the triple space $W_{0}$ is obtained from $W$ by appropriate blowups:

$$
W_{0}=\left[W ; B_{T}, S_{T} ; B_{F}, S_{F} ; B_{S}, S_{S} ; B_{C}, S_{C}\right] .
$$

Once again we obtain a manifold with corner. We label the hypersurfaces created by the blowups by $f F, f f, s f, c f$ respectively, and those from the lift by $f b, s b, c b$. The triple space $W_{0}$ is related to the double space $M_{0}^{2}$ by the $b$-fibrations

$$
\pi_{F}, \pi_{S}, \pi_{C}: W_{0} \rightarrow M_{0}^{2},
$$

corresponding to the projections $p_{F}, p_{S}, p_{C}: W \rightarrow Z$,

$$
\begin{aligned}
& p_{F}\left(t, t^{\prime}, m, m^{\prime}, m^{\prime \prime}\right)=\left(t^{\prime}, m^{\prime}, m^{\prime \prime}\right), \\
& p_{S}\left(t, t^{\prime}, m, m^{\prime}, m^{\prime \prime}\right)=\left(t-t^{\prime}, m, m^{\prime}\right), \\
& p_{C}\left(t, t^{\prime}, m, m^{\prime}, m^{\prime \prime}\right)=\left(t, t^{\prime}, m, m^{\prime \prime}\right) .
\end{aligned}
$$

Let $f: X \longrightarrow Y$ be a $b$-map between manifolds with corners, i.e., a $C^{\infty}$ map such that if $\rho_{i}^{\prime} \in C^{\infty}(Y), i=1, \ldots, N^{\prime}$ are defining functions for the boundary hypersurfaces of $Y$ and $\rho_{j} \in C^{\infty}(X), j=1, \ldots, N$. are defining functions for the boundary hypersurfaces of $X$ then

$$
f^{*} \rho_{i}^{\prime}=a_{i} \prod_{j=1} \rho_{j}^{k(i, j)}, 0<a_{i} \in C^{\infty}(X) .
$$

The non-negative integers $k(i, j)$ are the boundary exponents of $f$. In the following table we computed all boundary exponents of the $b$-fibrations $\pi_{F}, \pi_{S}, \pi_{C}$. 


\begin{tabular}{|l|l||l|l|l|l|l|l|l|}
\hline & & $\mathrm{fF}$ & $\mathrm{ff}$ & $\mathrm{sf}$ & $\mathrm{cf}$ & $\mathrm{fb}$ & $\mathrm{sb}$ & $\mathrm{cb}$ \\
\hline \multirow{4}{*}{$\pi_{F}$} & $\mathrm{ff}$ & 1 & 1 & 0 & 0 & 0 & 0 & 0 \\
\cline { 2 - 9 } & $\mathrm{lb}$ & 0 & 0 & 1 & 0 & 0 & 0 & 1 \\
\cline { 2 - 9 } & $\mathrm{rb}$ & 0 & 0 & 0 & 1 & 0 & 1 & 0 \\
\hline \multirow{4}{*}{$\pi_{S}$} & $\mathrm{ff}$ & 1 & 0 & 1 & 0 & 0 & 0 & 0 \\
\cline { 2 - 9 } & $\mathrm{lb}$ & 0 & 0 & 0 & 1 & 1 & 0 & 0 \\
\cline { 2 - 9 } & $\mathrm{rb}$ & 0 & 1 & 0 & 0 & 0 & 0 & 1 \\
\hline \multirow{3}{*}{$\pi_{C}$} & $\mathrm{ff}$ & 1 & 0 & 0 & 1 & 0 & 0 & 0 \\
\cline { 2 - 9 } & $\mathrm{lb}$ & 0 & 0 & 1 & 0 & 1 & 0 & 0 \\
\cline { 2 - 9 } & $\mathrm{rb}$ & 0 & 1 & 0 & 0 & 0 & 1 & 0 \\
\hline & $\nu$ & 0 & 0 & 0 & $\mathrm{n}+2$ & 0 & 0 & 0 \\
\hline
\end{tabular}

Also in the Table there is a 'density row', labelled ' $\nu$ ' which is important in the description of the composition results. These exponents are fixed by the natural identification of density bundles:

$$
\left(\pi_{F}\right)^{*} K D \otimes\left(\pi_{S}\right)^{*} K D \otimes\left(\pi_{C}\right)^{*}\left(K D^{\prime}\right) \otimes|d t|^{\frac{1}{2}} \cong \prod_{F=f F, f f, s f, c f, f b, s b, c b} \rho_{F}^{\nu_{F}} \cdot \Omega\left(W_{0}\right) .
$$

Here $K D^{\prime}$ is the half-density bundle with the opposite weighting to $K D$ so that $K D^{\prime} \otimes$ $K D \cong \Omega$.

Let $A \in \Psi_{h 0}^{-\infty, \mathcal{E}}\left(M ; \Omega^{\frac{1}{2}}\right), B \in \Psi_{h 0}^{-\infty, \mathcal{F}}\left(M, \Omega^{\frac{1}{2}}\right)$. The composition $C=A \circ B$ can be written in terms of their Schwartz kernels via the b-fibrations $\pi_{O}, O=F, S, C$ :

$$
\kappa_{C} K D^{\prime}=\left(\pi_{C}\right)_{*}\left[\left(\pi_{S}\right)^{*} \kappa_{A} \cdot\left(\pi_{F}\right)^{*} \kappa_{B} \cdot\left(\pi_{C}\right)^{*}\left(K D^{\prime}\right) \cdot\left(\tilde{\pi}_{C}\right)^{*}\left(\left|d t^{\prime}\right|^{\frac{1}{2}}\right)\right],
$$

where $\tilde{\pi}_{C}=\pi_{C}^{2} \circ \beta_{0}^{3}: W_{0} \rightarrow Z$ and $\beta_{0}^{3}$ is the blow down map.

By the push-forward theorem

$$
C \in \Psi_{h 0}^{-\infty, \mathcal{G}}\left(M, \Omega^{\frac{1}{2}}\right)
$$

for some index set $\mathcal{G}$. The index set $\mathcal{G}=\left(G_{f f}, G_{l b}, G_{r b}\right)$ can be computed by the Mellin transform

$$
\left\langle\kappa_{C} \cdot K D^{\prime}, \rho_{f f}^{z_{1}} \rho_{l b}^{z_{2}} \rho_{r b}^{z_{3}}\right\rangle=\left\langle\left(\pi_{F}\right)^{*}\left(\tilde{\kappa}_{A} \cdot\left(\pi_{S}\right)^{*}\left(\tilde{\kappa}_{B}\right) \cdot \prod_{F} \rho_{F}^{\nu_{F}} \cdot \Omega\left(W_{0}\right),\left(\pi_{C}\right)^{*}\left(\rho_{f f}^{z_{1}} \rho_{l b}^{z_{2}} \rho_{r b}^{z_{3}}\right)\right\rangle\right.
$$

where $\kappa_{A}=\tilde{\kappa}_{A} \cdot K D, \kappa_{B}=\tilde{\kappa}_{B} \cdot K D$. From the table we obtain the formulas for our index set. $\square$

3.4. Uniform structure of heat kernel. The proceeding construction enables us to prove the following theorem, which gives the uniform structure of the heat kernel for absolute (or relative) boundary condition.

THEOREM 3.3. If we denote by $e^{-t \Delta}$ the heat kernel of the Hodge Laplacian for the absolute (or relative) boundary condition, then

$$
e^{-t \Delta} \in \Psi_{h 0}^{2,2,0,0}(M, \Lambda M \otimes \xi) .
$$

Moreover the normal operators of $e^{-t \Delta}$ at the front face and the temporal face are explicitly computable.

Proof. Our proof is actually constructive. Namely we construct a (unique) $H \in$ $\Psi_{h 0}^{I}(M ; \Lambda M \otimes \xi)$ where $I=\{2,2,0,0\}$ such that

$$
t\left(\partial_{t}+\Delta\right) H=0 \bmod \Psi_{h 0}^{I^{\prime}}(M ; \Lambda M \otimes \xi)
$$


for $I^{\prime}=\{3,3,1,1\}$, and

$$
N_{h, 2}(H)=\mathrm{Id},
$$

and $\mathrm{H}$ satisfies the boundary condition

$$
P_{a}\left(\left.H\right|_{B_{l b}}\right)=0, P_{a}\left(\left.d H\right|_{B_{l b}}\right)=0 .
$$

Equations (3.3) and (3.17) translate to conditions on the normal operators of $H$ by using Proposition 3.1 :

$$
\begin{gathered}
\left({ }^{0} \sigma_{2}(t \Delta)-\frac{1}{2}(R+n)\right) N_{h, 2}(H)=0, \int_{\text {fiber }} N_{h, 2}(H)=\mathrm{Id} \\
\left(\partial_{T}+\Delta_{E}\right) N_{f, 2}(H)=0 .
\end{gathered}
$$

Finally the boundary condition translates into the same type boundary condition for (3.19).

The first equation is a fiber by fiber differential equation and can be solved uniquely subject to the integral condition. Furthermore because of the compatibility condition this fixes the integral conditions for (3.19). Thus the normal operator $N_{h, 2}(H)$ is necessarily the heat kernel for the elliptic boundary condition on the half tangent space. These two operators have the same indicial family, so using the existence part of the compatibility it follows that there is an element $G_{1} \in \Psi_{h 0}^{I}(M ; \Lambda M \otimes \xi)$ satisfying the symbolic conditions (3.18), (3.19).

This first approximation therefore satisfies

$$
t\left(\partial_{t}+\Delta\right) G_{1}=-R_{1}, R_{1} \in \Psi_{h 0}^{3,3,1,1}(M ; \Lambda M \otimes \xi) .
$$

Thus $G_{1}$ is already a parametrix. We now modify $G_{1}$. Using the heat calculus we can find a $G_{0} \in \Psi_{h 0}^{2,2, \infty, \infty}(M, \Lambda M \otimes \xi)$ such that

$$
t\left(\partial_{t}+\Delta\right) G_{0}=t \mathrm{Id}-t R_{0}
$$

where $R_{0} \in \Psi_{h 0}^{0, \infty, \infty, \infty}(M, \Lambda M \otimes \xi)$.

It follows that there is a correction term $G_{0}^{\prime} \in \Psi_{h 0}$ such that the modification of the parametrix $G_{2}=G_{1}-G_{0}^{\prime}$ still has the correct normal operator and is a parametrix in the strong sense that

$$
t\left(\partial_{t}+\Delta\right) G_{2}=t \mathrm{Id}-t R_{2}
$$

where $R_{2} \in \Psi_{h 0}^{\infty, 3,1,1}$.

By Proposition 3.2, $\left(R_{2}\right)^{k} \in \Psi_{h 0}^{\infty, 3 k, 1,1}$. Thus the Neumann series $\sum_{k=0}^{\infty}\left(R_{2}\right)^{k}$ can be summed modulo a term vanishing rapidly at $B_{f f}$, i.e. there exists $S^{\prime} \in \Psi_{h 0}^{\infty, 1,1}$ such that

$$
\left(I d-S^{\prime}\right)\left(\mathrm{Id}-R_{2}\right)=\mathrm{Id}-R_{3}, \quad R_{3} \in \Psi_{h 0}^{\infty, \infty, 1,1} .
$$

Thus Id $-R_{3}$ can be inverted with an operator of the same type. It follows that Id $-R_{2}$ has a two-side inverse Id $-S, S \in \Psi_{h 0}^{-\infty, 1,1}(M, F)$. This in turn means we have $\exp (-t \Delta)=G_{1}(I d-S)=G_{1}-G_{1} \circ S$ and we obtain our result. 
In the discussion below we will be concerned with the trace of the elements of $\Psi_{h 0}^{k, k}(M ; E)=\Psi_{h 0}^{k, k, 0,0}(M ; E)$. The elements of $\Psi_{h 0}^{k, k}(M ; E)$ are families of smoothing operators on $M$, hence trace class. By Lidsky's theorem the trace is the integral over the diagonal of the pointwise trace of the kernel. Consider the diagonal $\Delta(M) \times \mathbb{R}_{+}$ of $Z$, which lifts to an embedded submanifold of $M_{h 0}^{2}$. Let $i: \Delta_{h 0} \rightarrow M_{h 0}^{2}$ be the embedding of the lifted diagonal. We have

$$
\Delta_{h 0} \cong M_{h 0}=\left[M \times \mathbb{R}_{+} ; \partial M \times\{0\}, S\right]
$$

which blows down to $M \times \mathbb{R}_{4}$. Restricted to the lifted diagonal, the kernels of the elements of $\Psi_{h 0}^{k, k}(M ; E)$ can be interpreted as a density:

$$
\left.H o m(E) \otimes \Omega^{1 / 2}\left(M_{h 0}^{2}\right)\right|_{\Delta_{h 0}} \cong h o m(E) \otimes \Omega\left(M_{h 0}\right) \otimes \Omega^{\frac{1}{2}}\left(\mathbb{R}_{4}\right) .
$$

Thus the trace of $A \in \Psi_{h 0}^{2,2,0,0}(M ; E)$ is the push-forward to $\mathbb{R}_{+}$of the density

$$
\left.(\operatorname{tr} A)\right|_{\Delta_{h 0}} \in C^{\infty}\left(M_{h 0} ; \Omega\left(M_{h 0}\right) \otimes \Omega^{\frac{1}{2}}\left(\mathbb{R}_{+}\right)\right) .
$$

Lemma 3.4. As a map

$\operatorname{Tr}: \Psi_{h 0}^{k, k}(M ; E) \rightarrow t^{-\frac{n-k+2}{2}}\left(C^{\infty}\left(\mathbb{R}_{+}, \Omega^{\frac{1}{2}}\left(\mathbb{R}_{+}\right)\right)+t^{\frac{1}{2}} C^{\infty}\left(\mathbb{R}_{+}, \Omega^{\frac{1}{2}}\left(\mathbb{R}_{+}\right)\right)+O(t \log t)\right)$. i.e.

$$
\operatorname{Tr}(A)=t^{-\frac{n-k+2}{2}}\left(r_{A}(t)+t^{\frac{1}{2}} s_{A}(t)+O(t \log t)\right)|d t|^{\frac{1}{2}}
$$

where $r_{A}(t), s_{A}(t)$ are smooth functions of $t$. Moreover the leading terms can be computed by

$$
r_{A}(0)=\int_{M} \operatorname{tr}\left(N_{h, k}(A)\right), \quad s_{A}(0)=\int_{B_{f}} \operatorname{tr}\left(N_{f, k}(A)\right),
$$

where $M \rightarrow{ }^{0} T M$ is the 0 -section and $B_{f}$ is the front face of $M_{h 0}$.

Proof. This follows from the pushforward theorem, using Mellin transform. Namely, if we denote by $\beta^{\prime}$ the composition of the blowdown map $\beta_{1}: M_{h 0}=[M \times$ $\left.\mathbb{R}_{+} ; \partial M \times\{0\}, S\right] \rightarrow M \times \mathbb{R}_{+}$and the projection onto the second factor $M \times \mathbb{R}_{+} \rightarrow \mathbb{R}_{+}$, we have

$$
\left\langle\left(\beta^{\prime}\right)_{*}\left(\left.(\operatorname{tr} A)\right|_{\Delta_{h 0}}\right), t^{z}|d t|^{\frac{1}{2}}\right\rangle=\left\langle\left.(\operatorname{tr} A)\right|_{\Delta_{h 0}},\left(\beta^{\prime}\right)^{*}\left(t^{z}|d t|^{\frac{1}{2}}\right)\right\rangle
$$

Now

$$
\begin{aligned}
\left.K D\right|_{\Delta_{h 0}} \otimes\left(\beta^{\prime}\right)^{*}\left(|d t|^{\frac{1}{2}}\right) & =\left(\beta_{1}\right)^{*}\left(\left.t^{-\frac{n+2}{2}} \Omega^{\frac{1}{2}}\left(M^{2} \times \mathbb{R}_{+}\right) \otimes \Omega^{\frac{1}{2}}\left(\mathbb{R}_{+}\right)\right|_{\Delta\left(M \times \mathbb{R}_{+}\right)}\right) \\
& =\rho_{f f}^{-n} \rho_{t f}^{-n-1} \Omega\left(M_{h 0}\right),
\end{aligned}
$$

which gives us the desired result.

4. Clifford Structure and Cancellation. In order to prove our main result we need to modify our previous construction by incorporating the local index theorem type computation of Bismut-Zhang. A crucial ingredient is the Clifford structure of the exterior algebra, which we recall now. 
4.1. Clifford Structure of the Exterior Algebra. If $E$ is a finite dimenional vector space of dimension $n$, the exterior algebra $\Lambda\left(E^{*}\right)$ is naturally $\mathbb{Z}$-graded, which induces a natural $\mathbb{Z}_{2}$-grading. If $A \in \operatorname{End}\left(\Lambda\left(E^{*}\right)\right)$, we let $\operatorname{Tr}_{s}(A)$ denote the supertrace of $A$.

Now assume that $E$ is equipped with an inner product $g$. If $e \in E$, let $e^{*} \in E^{*}$ correspond to $e$ by the metric $g$. Set

$$
\begin{aligned}
& c(e)=e^{*} \wedge-i_{e} \\
& \hat{c}(e)=e^{*} \wedge+i_{e} .
\end{aligned}
$$

These define elements of $\operatorname{End}\left(\Lambda\left(E^{*}\right)\right)$. Moreover, we have the following commutator relations

$$
\begin{aligned}
& c(e) c\left(e^{\prime}\right)+c\left(e^{\prime}\right) c(e)=-2\left\langle e, e^{\prime}\right\rangle \\
& \hat{c}(e) \hat{c}\left(e^{\prime}\right)+\hat{c}\left(e^{\prime}\right) \hat{c}(e)=2\left\langle e, e^{\prime}\right\rangle \\
& c(e) \hat{c}\left(e^{\prime}\right)+\hat{c}\left(e^{\prime}\right) c(e)=0
\end{aligned}
$$

From (4.1), we see that $c$ and $\hat{c}$ extend to representations of the Clifford algebra of $E$. Furthermore, one verifies that $\operatorname{End}\left(\Lambda\left(E^{*}\right)\right)$ is generated as an algebra by 1 and the $c(e), \hat{c}(e)$ 's.

Let $e_{1}, \cdots, e_{n}$ be an orthonormal basis of $E$, and $e^{1}, \cdots, e^{n}$ the dual basis of $E^{*}$. A simple but essential algebraic fact is the following result which we quote from [BZ, Proposition 4.9].

Lemma 4.1. Among the monomials in the $c(e), \hat{c}(e)$ 's, only $c\left(e_{1}\right) \hat{c}\left(e_{1}\right) \cdots c\left(e_{n}\right) \hat{c}\left(e_{n}\right)$ has a nozero supertrace, which is

$$
\operatorname{Tr}_{s}\left[c\left(e_{1}\right) \hat{c}\left(e_{1}\right) \cdots c\left(e_{n}\right) \hat{c}\left(e_{n}\right)\right]=(-2)^{n} .
$$

4.2. Supertrace and Cancellation. The above discussion applied fiberwise to $T M$ shows that $\operatorname{End}\left(\Lambda^{*} M\right)$ is generated as an algebra by 1 and the $c(e), \hat{c}(e)$ 's for any local orthonormal basis $e$. This gives a natural filtration of $\operatorname{End}\left(\Lambda^{*} M\right)$, which we will exploit by making a global rescaling of $\operatorname{End}\left(\Lambda^{*} M \otimes \xi\right)$ near the front and temporal faces of $M_{h 0}^{2}$. Since this is localized near the diagonal, the vector bundle $\xi$ does not appear in the discussion and we happily suppress its presence.

Denote by

$$
F^{0} \subset F^{1} \subset \cdots \subset F^{n}
$$

the natural filtration on $\operatorname{End}\left(\Lambda^{*} M\right)$ induced by the Clifford structure introduced above, i.e., $F^{j}$ is the subspace generated by monomials of length $\leq j$ in $c(e), \hat{c}(e)$. Its associated graded algebra $G=\oplus G^{i}, G^{i}=F^{i} / F^{i-1}$, is naturally isomorphic to $\Lambda\left(T^{*} M\right) \otimes \Lambda\left(T^{*} M\right)$ (Cf. (4.1)). We distinguish the elements of the second copy of $\Lambda\left(T^{*} M\right)$ from the first by putting a hat on them. For example $\mathcal{R}=\frac{1}{8} R_{i j k l} e^{i} \wedge e^{j} \wedge$ $\hat{e}^{k} \wedge \hat{e}^{l} \in \Lambda\left(T^{*} M\right) \otimes \Lambda\left(T^{*} M\right)$.

Recall that the Berezin integral [BZ] takes elements of $\Lambda\left(T^{*} M\right) \otimes \Lambda\left(T^{*} M\right)$ and sends them to $\Lambda\left(T^{*} M\right)$ :

$$
\int^{B}: \Lambda\left(T^{*} M\right) \otimes \Lambda\left(T^{*} M\right) \rightarrow \Lambda\left(T^{*} M\right)
$$


The Berezin integral is intimately related to the supertrace and cancellation mechanism.

Applying the construction in $[\mathrm{DM}]$ we obtain a rescaled version of the pseudodifferential calculus constructed above, $\Psi_{h 0, G}^{I}\left(M ; \Lambda^{*} M \otimes \xi\right)$. For example, $\Psi_{h 0, G}^{2,2,0,0}\left(M ; \Lambda^{*} M \otimes \xi\right)$ consists of those elements of $\Psi_{h 0}^{2,2,0,0}\left(M ; \Lambda^{*} M \otimes \xi\right)$ which have the following asymptotic expansion near either the front or temporal face $F$ :

$$
k=\rho_{f f}^{2} \rho_{t f}^{2} \sum_{j=0}^{n} \rho_{F}^{\frac{j}{2}} k_{j}, \quad k_{j} \in C^{\infty}\left(M_{h 0}^{2}, F^{j} \otimes K D\right) .
$$

The normal operators $N_{h, k, G}, N_{f, k, G}$ of elements of $\Psi_{h 0, G}^{I}\left(M ; \Lambda^{*} M \otimes \xi\right)$ will now be taking values in the associated graded algebra $G \cong \Lambda\left(T^{*} M\right) \otimes \Lambda\left(T^{*} M\right)$.

The advantage of the modification comes from the following cancellation result. (Note the disappearance of the singular factor $t^{-\frac{n}{2}}$.)

LEMMA 4.2. As a map

$\operatorname{Tr}_{s}: \Psi_{h 0, G}^{k, k, 0,0}\left(M ; \Lambda^{*} M \otimes \xi\right) \rightarrow t^{\frac{k-2}{2}}\left(C^{\infty}\left(\mathbb{R}_{+}, \Omega^{\frac{1}{2}}\left(\mathbb{R}_{+}\right)\right)+t^{\frac{1}{2}} C^{\infty}\left(\mathbb{R}_{+}, \Omega^{\frac{1}{2}}\left(\mathbb{R}_{+}\right)\right)+O(t \log t)\right)$. i.e.

$$
\operatorname{Tr}_{s}(A)=t^{\frac{k-2}{2}}\left(r_{A}(t)+t^{\frac{1}{2}} s_{A}(t)+O(t \log t)\right)|d t|^{\frac{1}{2}}
$$

where $r_{A}(t), s_{A}(t)$ are smooth functions of $t$. Moreover the leading terms can be computed by

$$
r_{A}(0)=\int_{M} \int^{B} N_{h, k, G}(A), \quad s_{A}(0)=\int_{B_{f}} \int^{B} N_{f, k, G}(A) .
$$

Proof. This comes from the definition and the main algebraic fact Lemma 4.1.

With this in mind we have the following significant refinement on the structure of the heat kernel.

THEOREM 4.3. The heat kernel $e^{-t \Delta}$ of the Hodge Laplacian for the absolute (or relative) boundary condition, is an element of the rescaled heat calculus:

$$
e^{-t \Delta} \in \Psi_{h 0, G}^{2,2,0,0}\left(M, \Lambda^{*} M \otimes \xi\right) .
$$

Moreover the normal operators of $e^{-t \Delta}$ at the front face and the temporal face are given by

$$
\begin{aligned}
& N_{h, 2, G}=(4 \pi)^{-\frac{n}{2}} \exp \left(-|v|^{2}\right) \exp (-\mathcal{R}), \\
& N_{f, 2, G}=\exp \left(-T\left(\Delta_{E}+\mathcal{R}\right)\right),
\end{aligned}
$$

where $\mathcal{R}=\frac{1}{8} R_{i j k l} e^{i} \wedge e^{j} \wedge \hat{e}^{k} \wedge \hat{e}^{l}$ and $\Delta_{E}$ is the fibrewise Laplacian on the inward pointing half of tangent bundle $T M$ with the absolute (relative respectively) boundary condition.

Proof. We only need to adapt the previous argument to the rescaling. According to the Bochner-Weitzenböck formula,

$$
\Delta=\Delta^{c}-R_{i j k l} \operatorname{ext}\left(e_{i}\right) \operatorname{int}\left(e_{j}\right) \operatorname{ext}\left(e_{k}\right) \operatorname{int}\left(e_{l}\right) .
$$


Here $\Delta^{c}=\nabla^{*} \nabla$ is the connection Laplacian; with respect to any local orthonormal frame of $T M$ it is

$$
\Delta^{c}=-\sum_{i}\left(\nabla_{e_{i}}^{2}-\nabla_{\nabla_{e_{i}} e_{i}}\right)
$$

Expressing everything in terms of $c(e)$ and $\hat{c}(e)$, we have

$$
\Delta=\Delta^{c}+c(R)-\frac{1}{4} S
$$

with $S$ the scalar curvature and

$$
c(R)=\frac{1}{8} R_{i j k l} c\left(e_{i}\right) c\left(e_{j}\right) \hat{c}\left(e_{k}\right) \hat{c}\left(e_{l}\right) .
$$

Now since each Clifford element carries a weight of $\rho^{\frac{1}{2}}$ at both the temporal and the front faces, it follows then for $A \in \Psi_{h 0, G}^{I}\left(M ; \Lambda^{*} M \otimes \xi\right), t c(R) \circ A \in$ $\Psi_{h 0, G}^{I}\left(M ; \Lambda^{*} M \otimes \xi\right)$, and

$$
N_{h, G}(t c(R) \circ A)=\mathcal{R} N_{h, G}(A), \quad N_{f, G}(t c(R) \circ A)=T \mathcal{R} N_{f, G}(A) .
$$

Finally, for the connection, we note that

$$
\nabla_{e_{i}}=\Gamma_{i j}^{k} \operatorname{ext}\left(e_{k}\right) \operatorname{int}\left(e_{j}\right)=\frac{1}{4} \Gamma_{i j}^{k}\left(c\left(e_{k}\right)+\hat{c}\left(e_{k}\right)\right)\left(\hat{c}\left(e_{j}\right)-c\left(e_{j}\right)\right)
$$

again produces only lower order terms.

4.3. The Model Problem. Our model problem is to solve in $\mathbb{R}_{+}^{n}$ for the heat kernel of $\Delta+\mathcal{R}$, where $\mathcal{R}=\frac{1}{8} R_{i j k l} e^{i} \wedge e^{j} \wedge \hat{e}^{k} \wedge \hat{e}^{l}$, subject to the, say, absolute boundary conditions. More specifically, let $(x, y) \in \mathbb{R}_{+} \times \mathbb{R}^{n-1}=\mathbb{R}_{+}^{n}$ and $e_{0}=\partial_{x}$. Then we need to solve

$$
\left\{\begin{array}{l}
\left(\partial_{t}+\Delta+\mathcal{R}\right) K=0 \text { in } \mathbb{R}_{+}^{n} \\
\left.K\right|_{t=0}=\mathrm{I} \\
\left.e^{0} \wedge i_{e_{0}} K\right|_{x=0}=0 \\
\left.i_{e_{0}} e^{0} \wedge \frac{\partial K}{\partial x}\right|_{x=0}=0
\end{array}\right.
$$

LEMMA 4.4. Let $\mathcal{R}_{0}=\frac{1}{4} R_{0 j k l} e^{0} \wedge e^{j} \wedge \hat{e}^{k} \wedge \hat{e}^{l}$. We have

$$
\left[\mathcal{R}, i_{e_{0}} e^{0} \wedge\right]=\mathcal{R}_{0},\left[\mathcal{R}, e^{0} \wedge i_{e_{0}}\right]=-\mathcal{R}_{0}
$$

Proof. This is a straightforward computation using

$$
i_{e_{0}} e^{0} \wedge+e^{0} \wedge i_{e_{0}}=1 .
$$

In fact,

$$
\begin{aligned}
\mathcal{R}\left(i_{e_{0}} e^{0} \wedge\right) & =\left(\frac{1}{8} \sum_{i, j \neq 0} R_{i j k l} e^{i} \wedge e^{j} \wedge \hat{e}^{k} \wedge \hat{e}^{l}+\mathcal{R}_{0}\right)\left(i_{e_{0}} e^{0} \wedge\right) \\
& =\left(i_{e_{0}} e^{0} \wedge\right) \mathcal{R}+\mathcal{R}_{0}
\end{aligned}
$$


The other formula is obtained similarly.

Now, let

$$
\begin{aligned}
& K_{D}(t)=\frac{1}{(4 \pi t)^{n / 2}}\left(e^{-\left(x-x^{\prime}\right)^{2} / 4 t}-e^{-\left(x+x^{\prime}\right)^{2} / 4 t}\right) e^{-\left|y-y^{\prime}\right|^{2} / 4 t} \\
& K_{N}(t)=\frac{1}{(4 \pi t)^{n / 2}}\left(e^{-\left(x-x^{\prime}\right)^{2} / 4 t}+e^{-\left(x+x^{\prime}\right)^{2} / 4 t}\right) e^{-\left|y-y^{\prime}\right|^{2} / 4 t}
\end{aligned}
$$

be the Dirichlet and Neumann heat kernels respectively. Also, for $k=k\left(t, x, y, x^{\prime}, y^{\prime}\right)$, $\tilde{k}=\tilde{k}\left(t, x, y, x^{\prime}, y^{\prime}\right)$, their convolution is

$$
k * \tilde{k}=\int_{0}^{t} \int_{0}^{\infty} \int_{\mathbb{R}^{n-1}} k\left(s, x, y, x^{\prime}, y^{\prime}\right) \tilde{k}\left(t-s, x^{\prime}, y^{\prime}, x^{\prime \prime}, y^{\prime \prime}\right) d t d x^{\prime} d y^{\prime}
$$

Proposition 4.5. Let

$$
K_{0}=i_{e_{0}} e^{0} \wedge K_{N}(t) e^{-t \mathcal{R}}+e^{0} \wedge i_{e_{0}} K_{D}(t) e^{-t \mathcal{R}} .
$$

Then

$$
\left(\partial_{t}+\Delta+\mathcal{R}\right) K_{0}=\mathcal{R}_{0}\left[K_{N}(t)-K_{D}(t)\right] e^{-t \mathcal{R}} \equiv K_{1}
$$

And therefore, the solution of the model problem is

$$
K=K_{0}+K_{0} * K_{1}
$$

Proof. The first equation follows immediately from Lemma 4.4. Then we note that $K_{0}$ satisfies the absolute boundary condition and we apply the Duhamel's principle. The infinite sum terminates at the second term since $\mathcal{R}_{0}$ contains the nilpotent element $e^{0} \wedge$.

4.4. Proof of the Theorem. Finally we are in a position to prove Theorem 1.1. Proof of Theorem 1.1: As we mentioned before, we use the metric deformation (2.14) in the variation formula (2.11). To compute the right hand side of (2.11) we rewrite it as

$$
\lim _{t \rightarrow 0} \operatorname{Tr}_{s} F, F=*_{l}^{-1} \frac{\partial *_{l}}{\partial l} e^{-t \Delta_{l}}
$$

Since

$$
\begin{aligned}
*_{l}^{-1} \frac{\partial *_{l}}{\partial l} & =-\sum_{1 \leq i, j \leq n} \frac{1}{2}\left\langle\left(g_{l}^{-1} \frac{\partial g_{l}}{\partial l}\right)\left(e_{i}\right), e_{j}\right\rangle c\left(e_{i}\right) \hat{c}\left(e_{j}\right) \\
& =-h_{a b} c\left(e_{a}\right) \hat{c}\left(e_{b}\right) x+O\left(x^{2}\right) .
\end{aligned}
$$

(see [BZ, Proposition 4.15]), from Theorem 4.3 (and Theorem 3.3), we have

$$
F \in \Psi_{h 0, G}^{1,1,0,0}(M ; E) \text {. }
$$

Therefore, according to Lemma 4.2,

$$
\operatorname{Tr}_{s}(F)=t^{-\frac{1}{2}}\left(r(t)+t^{\frac{1}{2}} s(t)\right)|d t|^{\frac{1}{2}}
$$


with $r(0)$ and $s(0)$ given by (4.4).

Thus we must compute the two terms in (4.4). The first term computes the contribution from the interior of the manifolds and, just as in the case of the closed manifolds, can be seen to be zero. The term that really contributes to the variation formula is the second term which computes the boundary contribution. That is where the model problem comes in.

To compute the contribution of the model problem, we need to evaluate $K$ on the spatial diagonal and at $t=1$, and compute the corresponding Berezin integral, then integrate finally over $\mathbb{R}_{+} \times \partial M$. Restricted to the spatial diagonal and $t=1$, we have

$$
K=\frac{1}{(4 \pi)^{n / 2}} e^{-\mathcal{R}}+\frac{1}{(4 \pi)^{n / 2}} e^{-x^{2}} c\left(e_{0}\right) \hat{c}\left(e_{0}\right) e^{-\mathcal{R}}+\frac{1}{(4 \pi)^{(n-1) / 2}} f(x) \mathcal{R}_{0} e^{-\mathcal{R}}
$$

where

$$
f(x)=-\int_{0}^{1} \int_{0}^{\infty}\left(e^{-\left(x-x^{\prime}\right)^{2} / 4 s}+e^{-\left(x+x^{\prime}\right)^{2} / 4 s}\right) 2 e^{-\left(x+x^{\prime}\right)^{2} / 4(1-s)} d x^{\prime} d s
$$

Thus, we obtain

$$
\begin{aligned}
s(0)= & -\operatorname{rank} \rho \int_{\mathbb{R}_{+} \times \partial M} \int^{B} h_{a b} e^{a} \wedge \hat{e}^{b} x \frac{1}{(4 \pi)^{n / 2}} e^{-x^{2}} e^{0} \wedge \hat{e}^{0} \wedge e^{-\mathcal{R}} \\
& -\operatorname{rank} \rho \int_{\mathbb{R}_{+} \times \partial M} \int^{B} h_{a b} e^{a} \wedge \hat{e}^{b} x \frac{1}{(4 \pi)^{(n-1) / 2}} f(x) \mathcal{R}_{0} e^{-\mathcal{R}} .
\end{aligned}
$$

The first Berezin integral here can be explicitly evaluated:

$$
\begin{aligned}
& -\int_{\mathbb{R}_{+} \times \partial M} \int^{B} h_{a b} e^{a} \wedge \hat{e}^{b} x \frac{1}{(4 \pi)^{n / 2}} e^{-x^{2}} e^{0} \wedge \hat{e}^{0} \wedge e^{-\mathcal{R}} \\
= & \int_{\mathbb{R}_{+} \times \partial M} \frac{\partial}{\partial l} i^{*}\left(\tilde{e}\left(g_{0}, g_{l}\right)\right) e^{-x^{2}} x d x \\
= & \frac{\partial}{\partial l} \int_{\partial M} i^{*}\left(\tilde{e}\left(g_{0}, g_{l}\right)\right) .
\end{aligned}
$$

Taking $t$ to 0 and then integrating $l$ from 0 to 1 finally gives us the desired result.

\section{REFERENCES}

[BGV] N. Berline, E. Getzler, And M. Vergne, Heat Kernels and the Dirac Operator, Grundl. Der Math. Wiss. Band 298, Springer, Berlin, Heidelberg, New York, 1992.

[B] J.-M. BISMUT, From Quillen metrics to Reidemeister metrics: some aspects of the RaySinger analytic torsion, in Topological Method in Modern Mathematics, A Symposium in Honor of John Milnor's Sixtieth Birthday, Publish or Perish, 1993, pp. 273324.

[BF] J.-M. Bismut And D. Freed, The analysis of elliptic families, I., II., Comm. Math. Phys., 106 \& 107 (1986), pp. 159-176 \& 103-163.

[BGS] J.-M. Bismut, H. Gillet, And C. Soulé, Analytic Torsion and Holomorphic Determinant Bundles, I., II. III., Comm. Math. Phys., 115 (1988), pp. 49-78, 79-126, \& 301-351.

[BL] J.-M. Bismut and G. Lebeau, Complex Immersions and Quillen Metric, IHES Publ. Math., 74 (1991), pp. 1-298.

[BZ] J.-M. Bismut AND W. Zhang, An Extension of a Theorem by Cheeger and Müller, Astérisque 205, 1992.

[BG] T. BRANSON AND P. GILKEY, The Asymptotics of the Laplacian on a Manifold with Boundary, Comm. Partial Differential Equations, 15 (1990), pp. 245-272. 
[BFK] D. Burghelea, L. Friedlander, AND T. KAPPEler, Torsions for Manifolds with Boundary and Glueing Formulas, Math. Nachr., 208 (1999), pp. 31-91.

[BFKM] D. Burghelea, L. Friedlander, T. Kappeler, AND P. MCDonald, Analytic and Reidemeister torsion for representations in finite type Hilbert modules, Geom. Funct. Anal., 6 (1996), pp. 751-859.

[C] J. Cheeger, Analytic Torsion and the Heat Equation, Ann. Math., 109 (1979), pp. 259322.

[DO] S. K. Donaldson, Infinite determinants, stable bundles and curvature, Duke Math. J., 54 (1987), pp. 231-247.

[D1] X. DAI, Adiabatic limits, the non-multiplicativity of signature and Leray spectralsequence, Journal of A. M. S., 4 (1991), pp. 265-321.

[D2] X. DAI, Geometric Invariants and Their Adiabatic Limits (part II), Proc. Symposia Pure Math., 54 (1993), pp. 145-156.

[DM] X. DAI AND R. B. Melrose, Adiabatic limit of analytic torsion, preprint.

[F] W. Franz, Über die Torsion einer überdeckrung, J. Reine Angew. Math., 173 (1935), pp. 245-254.

[G] E. Getzler, A Short Proof of the Atiyah-Singer Index Theorem, Topology, 25 (1986), pp. 111-117.

[GS] H. GILlET AND C. SoulÉ, An arithmetic Riemann-Roch theorem, Invent. Math., 110 (1992), pp. 473-543.

[LR] J. Lott AND M. Rothenberg, Analytic Torsion for Group Actions, J. Diff. Geom., 34 (1991), pp. 431-481.

[L] W. LücK, Analytic and topological torsion for manifolds with boundary and symmetry, J. Diff. Geom., 37 (1993), pp. 263-322.

[LS] W. LÜCK AND T. SCHICK, $L^{2}$-torsion of hyperbolic manifolds of finite volume, preprint.

[MS] H. McKean AND I. M. Singer, Curvature and the eigenforms of the Laplacian, J. Diff. Geom., 1 (1967), pp. 43-69.

[M1] R. B. MELrose, The Atiyah-Patodi-Singer index theorem, Research Notes in Mathematics 4, A. K. Peters, Massachusatts, 1993.

[M2] R. B. MELRose, Differential calculus on manifolds with corners, in preparation.

[M3] R. B. MELrose, Pseudo-differential operators, corners and singular limits, in Proceedings of ICM Kyoto, 1990, pp. 217-234.

[Mü1] W. MüLLER, Analytic torsion and R-torsion of Riemannian manifolds, Adv. in Math., 28 (1978), pp. 233-305.

[Mü2] W. MülLER, Analytic Torsion and R-torsion for Unimodular Representations, J. Amer. Math. Soc., 6 (1993), pp. 721-753.

[Q1] D. QUILLEN, Superconnections and the Chern character, Topology, 24 (1985), pp. 89-95.

[Q2] D. QUILLEN, Determinants of Cauchy-Riemann operators over a Riemann surface, Funct. Anal. Appl., 14 (1985), pp. 31-34.

[RS1] D. RAY AND I. M. SINGER, R-torsion and the Laplacian on Riemannian manifolds, Adv. in Math., 7 (1971), pp. 145-210.

[RS2] D. RAY AND I. M. Singer, Analytic torsion for complex manifolds, Ann. Math., 98 (1973), pp. 154-177.

[R] K. REIDEMEISTER, Homotopieringe und Linsenraüm, in Hamburger Abhandl. 11, 1935, pp. 102-109.

[V] S. M. VISHIK, Generalized Ray-Singer conjecture I: a manifold with a smooth boundary, Comm. Math. Phys., 167 (1995), pp. 1-102.

[W] E. WITten, Quantum field theory and Jones polynomial, Comm. Math. Phys., 121 (1989), pp. 351-399. 